\title{
On the General Properties of Steady Gravitational Thermal Flows of Liquid Metals in Variable Cross-section Containers
}

\author{
Marcello Lappa*1, Hermes Ferialdi ${ }^{1}$ \\ ${ }^{1}$ Department of Mechanical and Aerospace Engineering, University of Strathclyde, James Weir Building, 75 \\ Montrose Street, Glasgow, G1 1XJ, UK \\ ${ }^{* 1}$ marcello.lappa@strath.ac.uk
}

\begin{abstract}
Thermogravitational flows of liquid metals in open or closed ducts and containers play a relevant role in a variety of applications in mechanical, materials and nuclear engineering. Such flows are known to be very sensitive to the effective shape of the container used to host the fluid and its thermal boundary conditions. For the case of temperature gradients having the main component directed along the horizontal direction, related convective phenomena fall under the general heading of "Hadley flow". Here we introduce a general framework for the determination of the properties of these flows in the case of domains having converging or diverging top and bottom walls. The framework is built via a hybrid approach in which typical techniques of CFD a re used in synergy with analytical solutions of the energy equation. The proper use of initial and boundary conditions results in algorithm convergence acceleration. The role played by the top and bottom wall inclination with respect to the horizontal is assessed through parametric investigation.
\end{abstract}

\section{Keywords}

Thermal Convection; Liquid Metal Flow; Navier-Stokes Equations; Analytic; Numerical Solutions

\section{Introduction}

Thermogravitational flow is classical subject of investigation in nonlinear dynamics. The related various buoyancy mechanisms in cavities and layers (for different heating and boundary conditions) are often used as archetypal systems in the study of complex phenomena because these configurations are highly relevant to a number of engineering applications.

As an example, such flows typically play an important role in the solidification of industrial castings and ingots. They can have remarkable consequences on the quality and mechanical resistance of such products due to the formation of "defects" such as freckles and channels in the final structure of the material (Ludwig et al., 2004; Abhilash et al., 2005).

Similar considerations can be applied to the companion field of "crystal grow th from the melt", which is typically concerned with the production of high quality crystals of metallic (e.g., semiconductor or superconductor) materials starting from the corresponding liquid state (e.g., the horizontal Bridgman (HB), the Czochralski (CZ) or the Floating zone (FZ) technique). Though such production methods differ in the shape of the container used to host the liquid and/or the direction of the applied temperature gradient (Lappa, 2005), the presence of convection of gravitational (buoyancy) nature in the melt cannot be avoided. Such flows can adversely affect the perfection and purity of the ordered crystalline structures of the solidified material (bulk convection typically leads to the formation of "striations" or "segregations", at the micro or macro scale, respectively; Dupret and Van der Bogaert, 1994; Monberg, 1994).

Additional interest for such flows originates from their relevance to the nuclear engineering area. As an example, a new class of advanced-type nuclear devices (liquid metal fast reactor or LMFR) is being exploited where water as the primary coolant has been replaced by a liquid metal. Because a metal has obviously a much higher heat capacity and thermal conductivity than water, it can be used to remove heat more efficiently. This makes the use of 
liquid metals as primary coolants very convenient in situations where limiting the size of the plant is a factor of crucial importance (Zrodnikov et al., 2004).

In all these circumstances, obviously, having the capability to assess in advance the intensity and structure of such flows might be regarded as the necessary basis to improve existing methods developing new optimisation strategies.

As already discussed in Lappa (2012a), determining solutions of the equations governing such flows (NavierStokes + energy equations) in analytic form is an extremely challenging task. These exact solutions are known to exist only in very special circumstances (most of which being purely "ideal") such as domains infinitely extended along the direction of the imposed temperature gradient or flows attaining a purely "parallel state" (that is a kind of motion for which only one of the two velocity components in the plane containing the temperature gradient is not zero).

The present investigation proceeds one step further and addresses the specific case in which the extension of the domain along the horizontal direction is finite and the walls bounding the liquid from above and from below are not horizontal, thereby paving the way to a new category of studies devoted to the analysis of the strength and topology of thermogravitational flows of liquid metals in "converging or diverging" containers or in geometries $w$ ith inclined $w$ alls or inclined temperature gradient. Existing studies on such subjects are relatively limited (Hong et al., 1993; Delgado-Buscalioni and Crespo del Arco., 2001;Lappa, 2009 and 2012b).

Such a new framew ork is built via a hybrid approach in which typical techniques of CFD are used in synergy with analytical solutions of the energy equation.

\section{Geometry and Mathematical Model}

\section{The Geometry}

For concreteness, initially we consider a two-dimensional shallow cavity symmetric with respect to the horizontal direction with average depth $d$, laterally delimited by solid walls at different temperatures (one cooled, the other heated) having height dhot and dcold such that $d=($ dhot $+d c o l d) / 2$. The overall system aspect ratio (A) is defined as its length-to-average-depth ratio $(A=L / d)$. Another relevant characteristic geometrical parameter (refer to Fig. 1) is the expansion (compression) ratio $\eta=\mathrm{dhot} / \mathrm{dcold}$, which can be $>1$ (diverging geometry) or $<1$ (converging geometry), while for $\eta=1$ one would recover the classical case with horizontal boundaries originally studied by Hadley (1735).

The top and bottom walls are assumed to be adiabatic (no heat exchange).
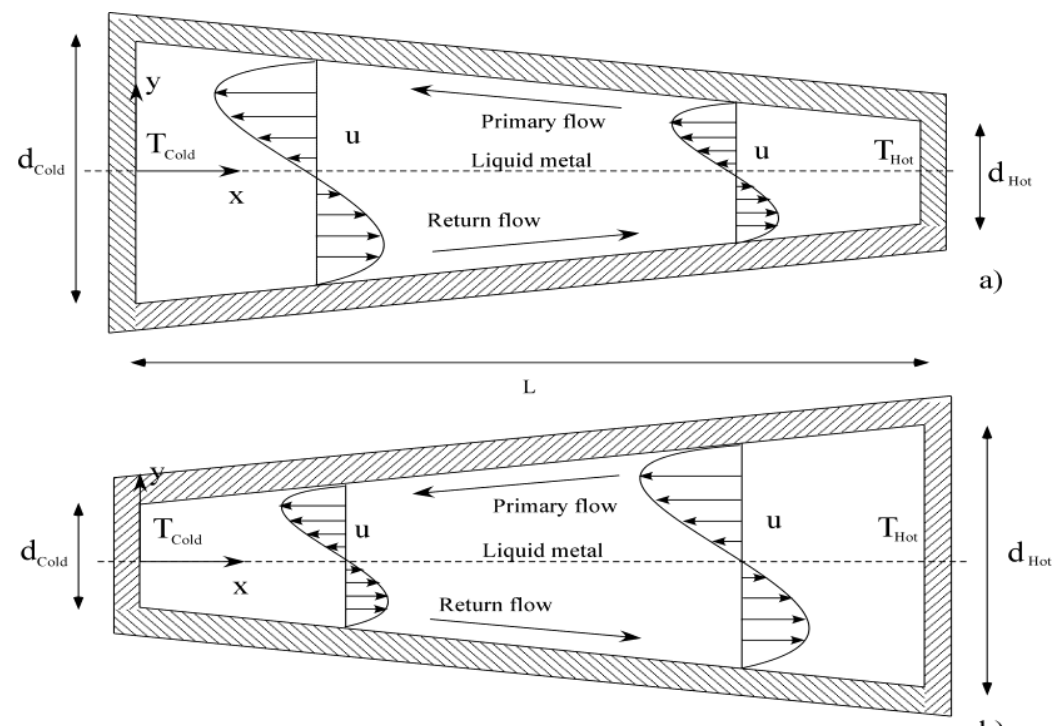

b)

FIG.1 SKETCH OF THE CONSIDERED GEOMETRY AND RELATED THERMAL AND KINEMATIC BOUNDARY CONDITIONS:a) $\eta<1$, b) $\eta>1$. 


\section{Governing Equations and Parameters}

The Rayleigh number is defined as $\operatorname{Ra}=\operatorname{Gr} \operatorname{Pr}=g \beta T \Delta T d 3 / v \alpha$ where $\Delta \mathrm{T}$ is the horizontal temperature difference, $\mathrm{d}$ is the average distance between the top and bottom boundaries, $\alpha$ is the thermal diffusivity, $v$ is the kinematic viscosity, $\beta$ T is the thermal expansion coefficient, Pr the ratio between $v$ and $\alpha$, and Gr is the Grashof number. Referring velocity and temperature to the scales $\alpha / \mathrm{d}$ and $\Delta \mathrm{T}$, respectively and scaling all distances on $\mathrm{d}$, the governing equations in nondimensional form (incompressible form) read

$$
\begin{gathered}
\underline{\nabla} \cdot \underline{V}=0 \\
\frac{\partial \underline{V}}{\partial}=-\underline{\nabla} p-\underline{\nabla} \cdot[\underline{V} \underline{V}]+\operatorname{Pr} \nabla^{2} \underline{V}-\operatorname{Pr} R a T \underline{i}_{g} \\
\frac{\partial T}{\partial}+\underline{\nabla} \cdot[\underline{V} T]=\nabla^{2} T
\end{gathered}
$$

where $\mathrm{V}, \mathrm{T}$ and $\mathrm{p}$ are the nondimensional velocity, temperature and pressure, respectively, ig is the unit vector along the direction of gravity and the Boussinesq approximation has been used for the buoyancy production term in the momentum equation.

A distinguishing mark of liquid metals with respect to other substances (such as water or oils) is the relatively small value attained by the Prandtl number ( $\operatorname{Pr}>1$ for water or oils, $\operatorname{Pr}<<1$ for liquid metals). Her e we concentrate on a "representative" liquid metal for which $\operatorname{Pr}=0.01$.

\section{Results}

\section{Analytic Solutions for Quiescent Conditions}

Before starting to deal with the nature and structure of the emerging flow for finite values of the Rayleigh number, it is instructive to consider the behaviour of the temperature field in the limit as $\mathrm{Ra} \rightarrow 0$, namely conditions of creeping flow or quiescent state. For such a case, indeed, though the assumption of horizontal parallel top and bottom boundaries is removed, an analytic solution can be still found.

Our interest in such an expression stems from a two-fold purpose. First it can be used to show that the assumption of linear temperature increase along the horizontal ( $x$ axis) is no longer valid. This assumption is generally invoked for differentially heated rectangular enclosures with adiabatic top and bottom walls in quiescent (no flow) conditions or even in non-quiescent conditions for liquid metals provided one of the two following conditions (or both) is satisfied: $\operatorname{Pr} \rightarrow 0$ or the flow is parallel, i.e. the considered system has an infinite extension along the horizontal direction.

Second, such an analytical expression can be used as a relevant initial condition to accelerate the convergence of the algorithm relating to the numerical solution of the momentum and mass conservation equations in the more general case for which both A, Pr and Ra have finite values and $\eta \neq 1$.

Most conveniently, we start from the balance of energy cast in its simplest form, that is, the conservation of energy flux along the horizontal direction:

$$
d(x) \frac{d T}{d x}=c_{1}
$$

where $\mathrm{d}(\mathrm{x})$ is the nondimensional extension of the generic cross section, which in turn can be expressed as:

$$
d(x)=d_{\text {cold }}+\left[\frac{d_{\text {hot }}-d_{\text {cold }}}{A}\right] x=d_{\text {cold }}\left[1+(\eta-1) \frac{x}{A}\right]
$$

Substituting eq. (5) into eq. (4) gives:

$$
\frac{d T}{d x}=\frac{c_{1}}{d_{\text {cold }}[1+(\eta-1) x / A]}
$$

which, once integrated, yields: 


$$
T(x)=\frac{c_{1} A}{d_{\text {cold }}(\eta-1)} \ln [1+(\eta-1) x / A]+c_{2}
$$

The two constants $c_{1}$ and $c_{2}$ can be determined forcing eq. (7) to satisfy the boundary conditions at the two sidew alls, namely:

$$
\begin{array}{ll}
\mathrm{x}=0 & \mathrm{~T}=0 \rightarrow c_{2}=0 \\
\mathrm{x}=\mathrm{A} & \mathrm{T}=1 \rightarrow c_{1}=\frac{d_{\text {cold }}(\eta-1)}{A \ln (\eta)}
\end{array}
$$

Accordingly, eq.(7) can be cast in compact form as:

$$
T(x)=\frac{\ln [1+(\eta-1) x / A]}{\ln (\eta)}
$$

where, obviously:

$$
\lim _{\eta \rightarrow 1} \frac{\ln [1+(\eta-1) x / A]}{\ln (\eta)}=\frac{x}{A}
$$

Equation (9) plotted in Fig. 2 for different values of the parameter $\eta$, clearly shows the departure of the temperature profile from the purely linear distribution theoretically established for $\eta=1$. The resulting profile is concave or convex for $\eta<1$ or $\eta>1$, respectively. As anticipated, such behaviours should be regarded as an evident difference with respect to past situations in which the flow was assumed to be purely parallel or "purely thermally diffusive" conditions were considered in finite-size rectangular enclosures (for very small values of the Prandtl number such as those which are typical of liquid metals $(\operatorname{Pr}<<1)$ and for relatively small values of the control parameter Ra).

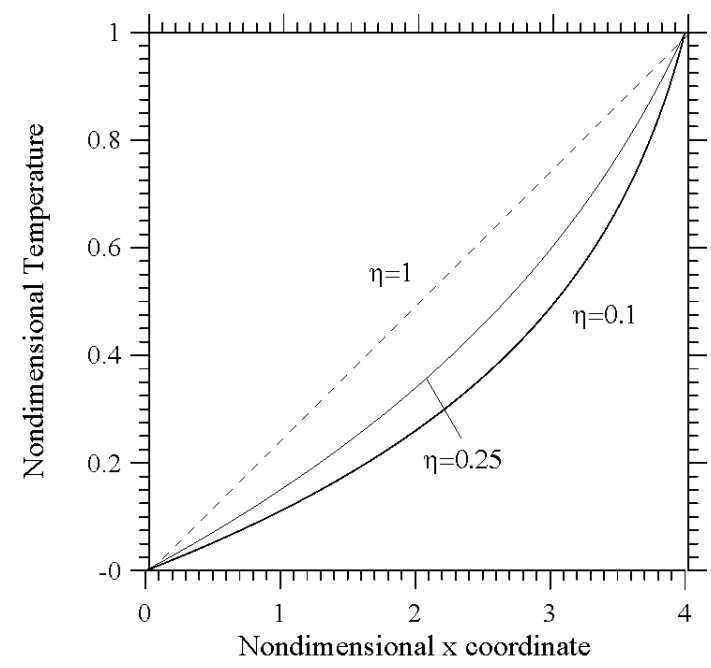

a)

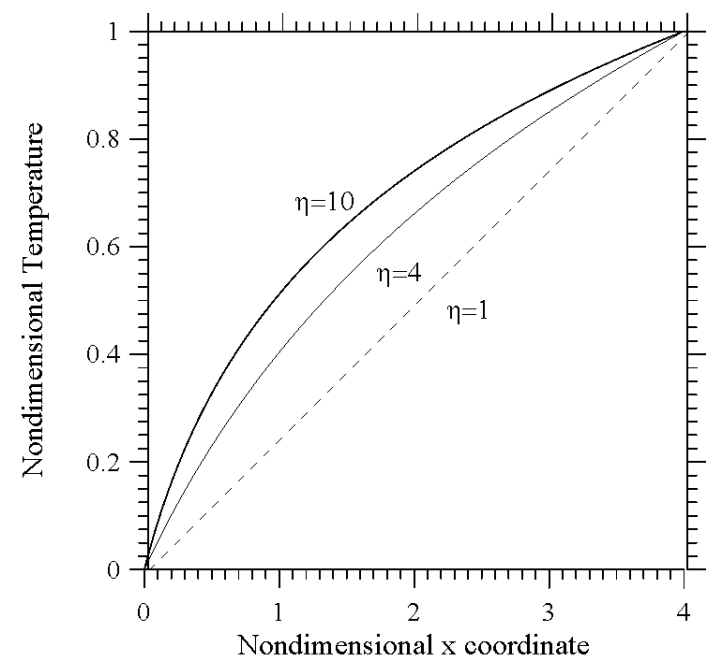

b)

FIG .2 EXACT SOLUTION FOR THE TEMPERATURE DISTRIBTION FOR A=4 AND Ra=0 (A DASHED LINE IS USED FOR THE CORRESPONDING IDEAL LINEAR TEMPERATURE PROFILE): a) $\eta<1$ (CONVERGING WALLS) ; b). $\eta>1$ (DIVERGING WALLS)

\section{Numerical Results}

The governing equations (1-3) have been solved in their complete non-linear and time-dependent form using a projection method, that is an approach by which the computation of the velocity field is articulated into two main macro steps providing separately an intermediate field that has the correct content of vorticity but does not satisfy the continuity equation and a final velocity field, which has both the correct values of divergence and curl. Such a field is "unique" according to the Ladyzhenskaya decomposition theorem (Ladyzhenskaya, 1969), the reader being referred to various books in the literature for additional details about this well-known approach (Fletcher, 1991).

The open source software Open Foam has been used for the computations. For each aspect ratio a mesh refinement study has been conducted in advance in order to determine the minimum number of computational points 
required to get grid-independent solutions in the case of uniform grids ( $\mathrm{NxxNy}=100 \times 30$ and 200x30 for $\mathrm{A}=4$ and $A=10$, respectively). The typical structure of buoyancy flow is shown in Fig. 3 for different values of the aspect ratio and of the parameter $\eta$. All cases correspond to subcritical conditions, namely circumstances for which the asymptotic state attained by thermogravitational flow is steady. The governing equations have been solved assuming no-slip conditions on the walls, conditions of prescribed temperature at the left and right sidewalls and adiabatic top and bottom (inclined) boundaries.

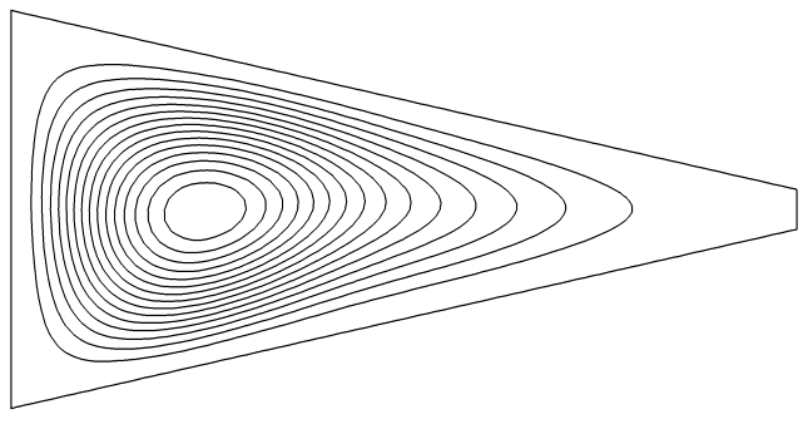

a)

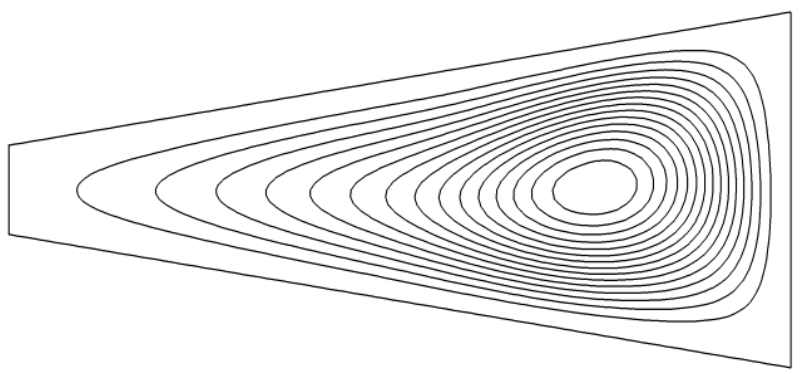

b)

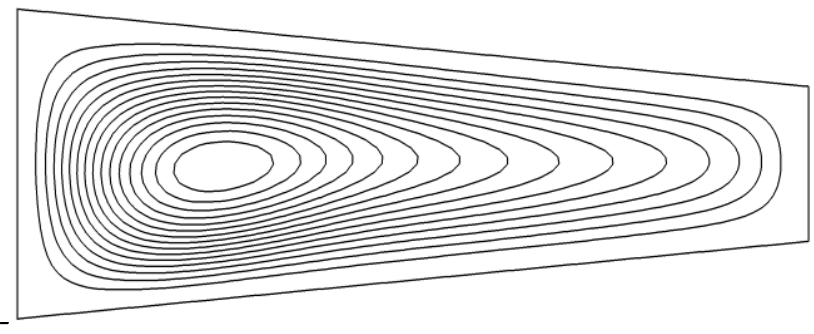

c)

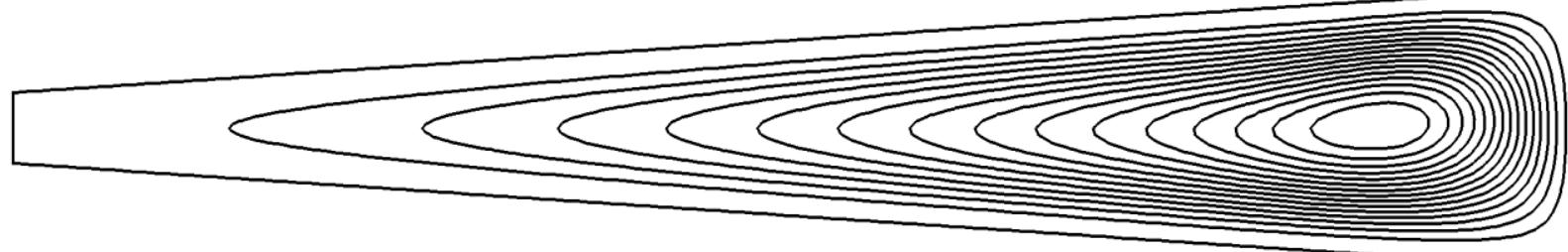

d)

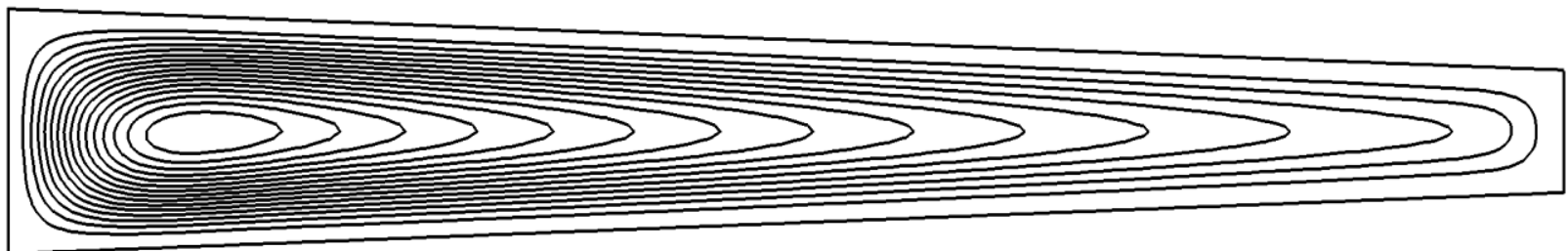

e)

FIG. 3 STEADY VELOCITY FIELD (STREAMLINES)FOR Ra=100 AND DIFFERENT VALUES OF THE ASPECTRATIO AND $\eta:$ a) A = $4 \mathrm{AND} \eta=0.1, \mathrm{~b}) \mathrm{A}=4$ AND $\eta=4, \mathrm{c}) \mathrm{A}=4$ AND $\eta=0.5$, d) $\mathrm{A}=10$ AND $\eta=4, \mathrm{e}) \mathrm{A}=10$ AND $\eta=0.5$. 


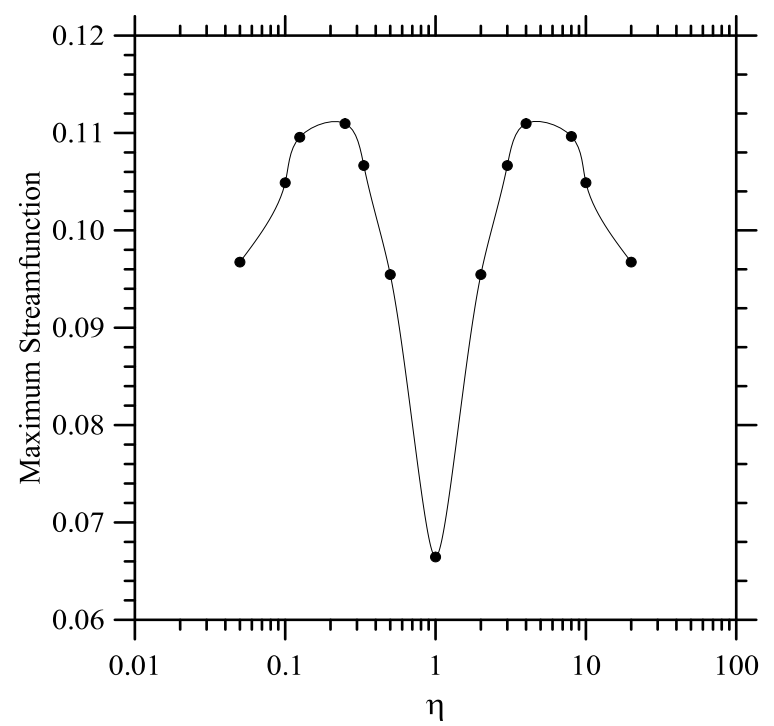

a)

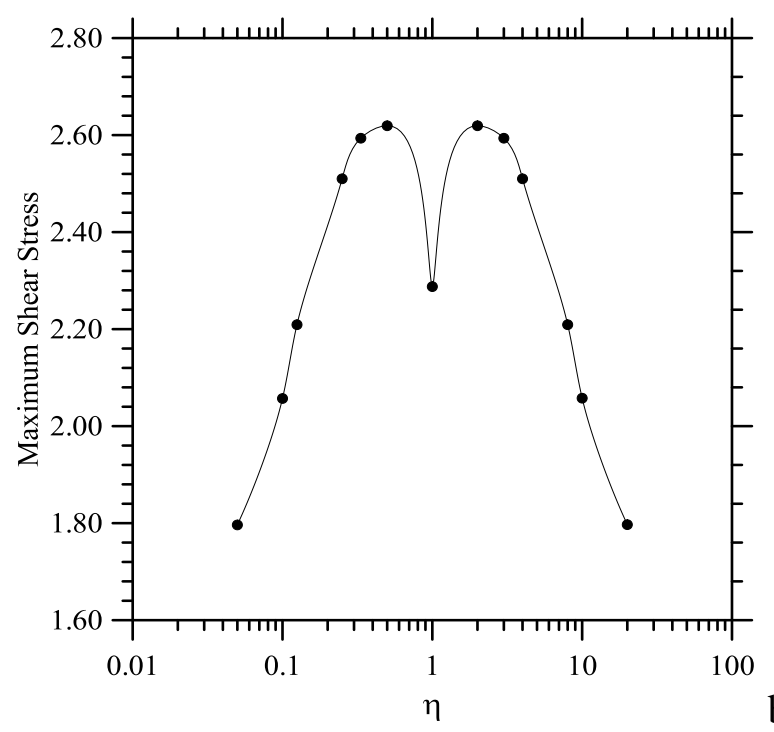

b)

FIG. 4 NONDIMENSIONAL MAXIMUMSTREAMFUNCTION (a) ANDSHEARSTRESS (b) A FUNCTION OF THE PARAMETER $\eta$ FOR A=4

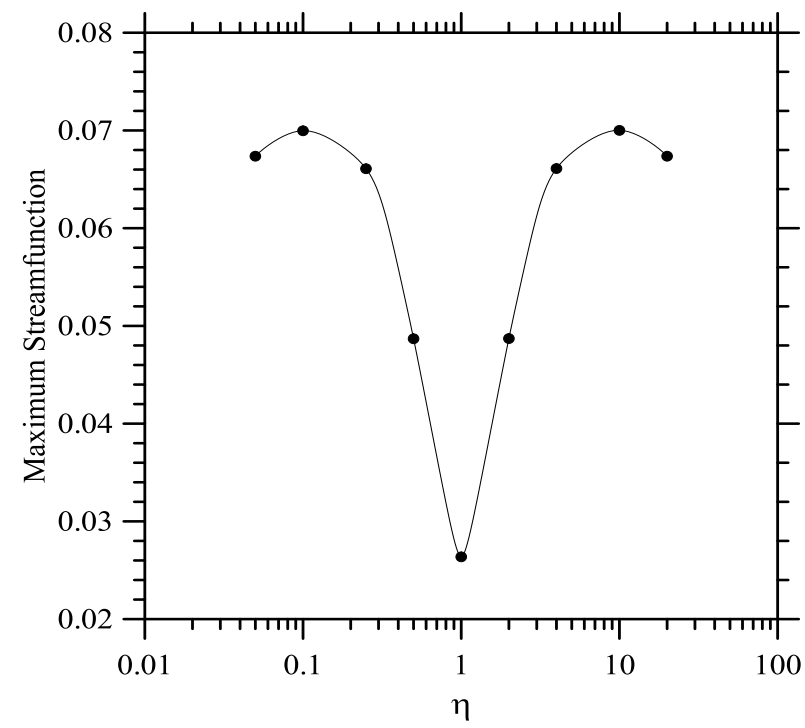

c)

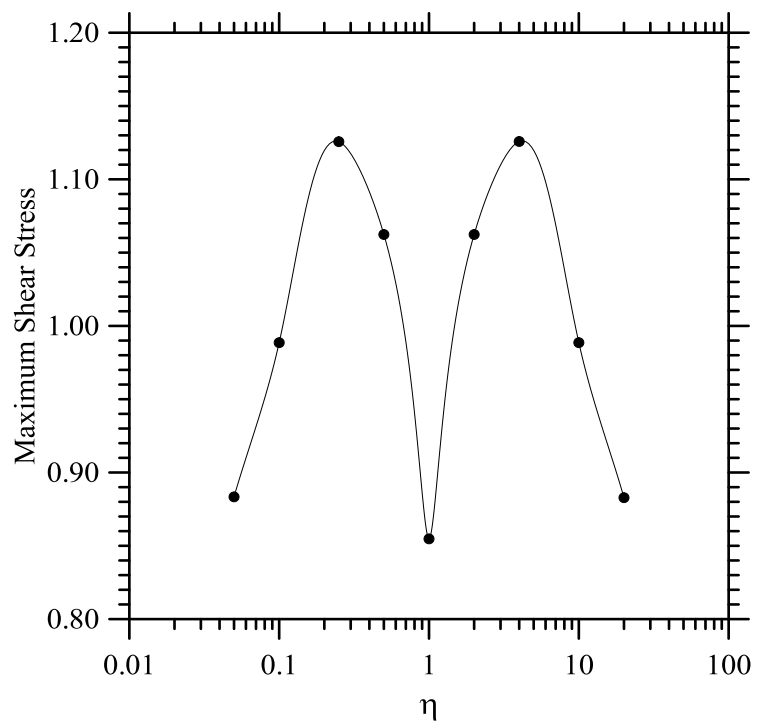

d)

FIG. 5 NONDIMENSIONAL MAXIMUMSTREAMFUNCTION (a) ANDSHEARSTRESS (b)AS A FUNCTION OF THE PARAMETER $\eta$ FOR $\mathrm{A}=10$.

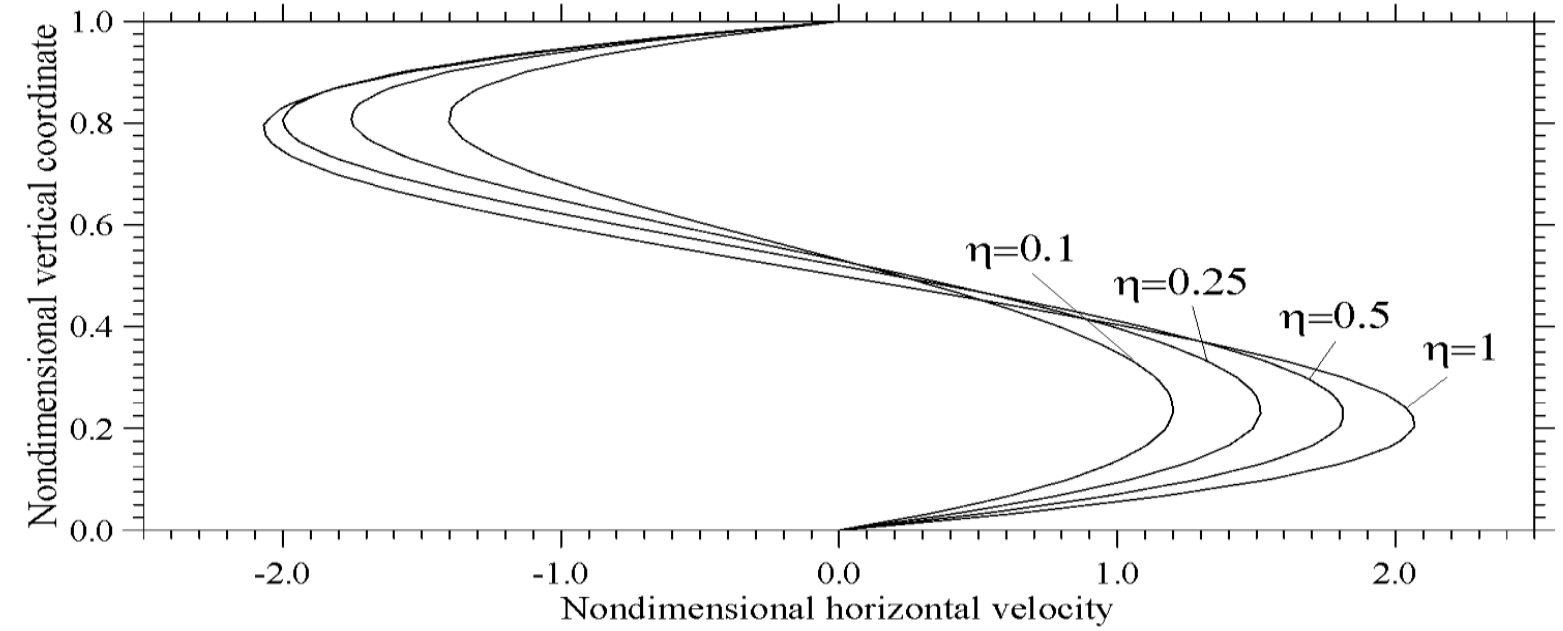

FIG. 6 NONDIMENSIONAL HORIZONTAL VELOCITY U AS A FUNCTION OF THE VERTICALCOORDINATEY AT x=A/2 FOR A=4 AND DIFFERENT V ALUES OF THE PARAMETER $\eta$. 
Figures 4 and 5 shed some additional light on the problem considered illustrating the non-trivial dependence of the flow strength (in terms of maximum value attained by the streamfunction) and the (maximum) shear stress ( $\partial \mathrm{u} / \partial \mathrm{y}$ where $u$ is the horizontal component of velocity and $y$ the vertical coordinate) on the parameter $\eta$. As shown in such figures, both the streamfunction and the nondimensional shear stress attain a minimum when the walls are perfectly horizontal, i.e. for $\eta=1$. This case has been already extensively investigated in the literature (the reader being referred, e.g., to the excellent numerical study by Gelfgat et al., 1999). Any departure from this ideal condition, however, causes an increase in the shear stress and $\psi$. The related dependence is relatively complex (non-monotone) as witnessed by the existence of two relative maxima. These can be seen in the shear stress curve for $\eta=0.5$ and $\eta=2$ for $A=4$ and $\eta=0.25$ and $\eta=4$ for $A=10$. Similarly, for the stream function the maxima are located at $\eta=0.25$ and $\eta=4$ for $A=4$ and $\eta=0.1$ and $\eta=10$ for $A=10$. Both quantities display a symmetric distribution with respect to the situation of horizontal top and bottom walls (the plots in logarithmic scale are mirror symmetric with respect to $\eta=1)$.

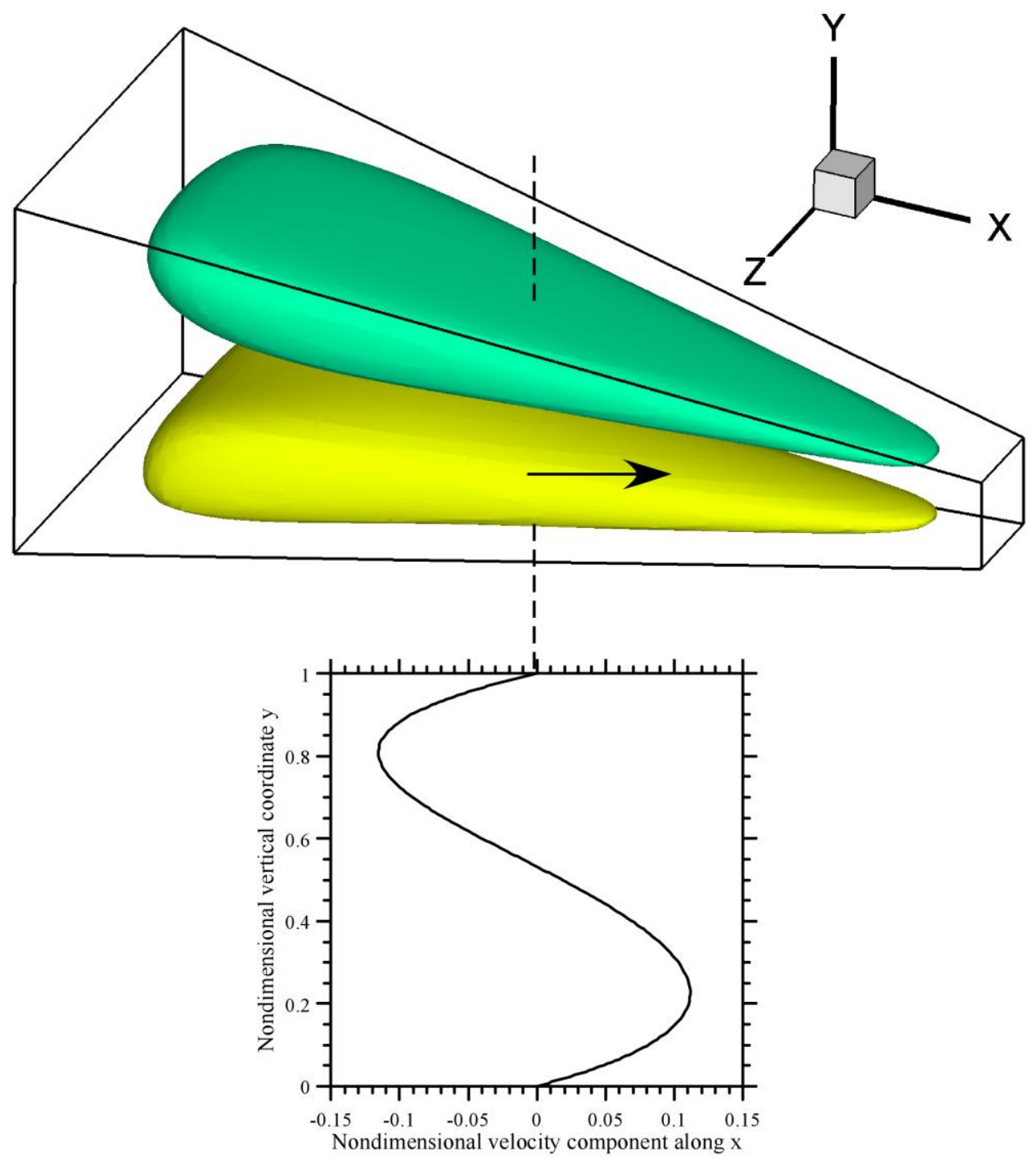

FIG. 7 STEADY V ELOCITY FIELD (ISOSURFACES U)FOR A=4, Ra=100 AND $\eta=1 / 16$ (3D SIMULATION FOR A CONTAINER HAVING THE SHAPE OF A FRUSTUM OF PYRAMID, NxxNyxNz=100x30x30,MAXIMUM SHEAR $\partial \mathrm{u} / \partial \mathrm{y}=1.3)$ ANDU VELOCITY PROFILE AT $x=A / 2$ AND

$\mathrm{z}=0$

Figure 6, provides some information about the existence of "inflection points" in the velocity profile.

Such points are known to play a crucial role in determining the "future" behaviour of the system when the intensity of the driving force is increased. For purely parallel flow, indeed, it is known that in the limit as Pr $\rightarrow 0$ the following (Rayleigh's) theorem holds: In a shear flow a necessary condition for instability is that there must be a point of inflection in the velocity profile $\mathrm{u}=\mathrm{u}(\mathrm{y})$, i.e. a point where $\mathrm{d} 2 \mathrm{u} / \mathrm{dy} 2=0$. As illustrated by Tollmien (1936), 
this theorem can be regarded as also a sufficient condition in many situations. For more comprehensive discussions the interested readers may consider Lin (1944), Rosenbluth and Simon (1964), Drazin and How ard (1966).

According to Figure 6, though in our case the flow is not parallel, inflection points in the profile of horizontal velocity u can be still identified.

Similar conclusions can be applied to fully three-dimensional (3D) configurations having a frustum-of-pyramid shape (for which we performed a limited set of simulations using yet eq. (9) as initial condition, the parameter $\eta$ appearing there, being this time the ratio between the areas of the two end walls). Such results, shown, e.g., in Fig. 7 for the case $A=4$ and $\eta=1 / 16$, confirm that, though the maximum of the shear stress $\partial \mathrm{u} / \partial \mathrm{y}$ experiences a decrease with respect to the corresponding $2 \mathrm{D}$ case $(\partial \mathrm{u} / \partial \mathrm{y} \cong 1.3$ for the $3 \mathrm{D}$ case to be compared with $\partial \mathrm{u} / \partial \mathrm{y} \cong 1.84$ for the $2 \mathrm{D}$ case having the same value of $\eta$ ), the existence of inflection points in the distribution of horizontal velocity $u$ is retained.

Furthermore, inflection points can be also found in the profile of the other horizontal velocity component pertaining to the $3 \mathrm{D}$ simulations, namely the component $\mathrm{w}$ along the $\mathrm{z}$ axis (Fig. 8).

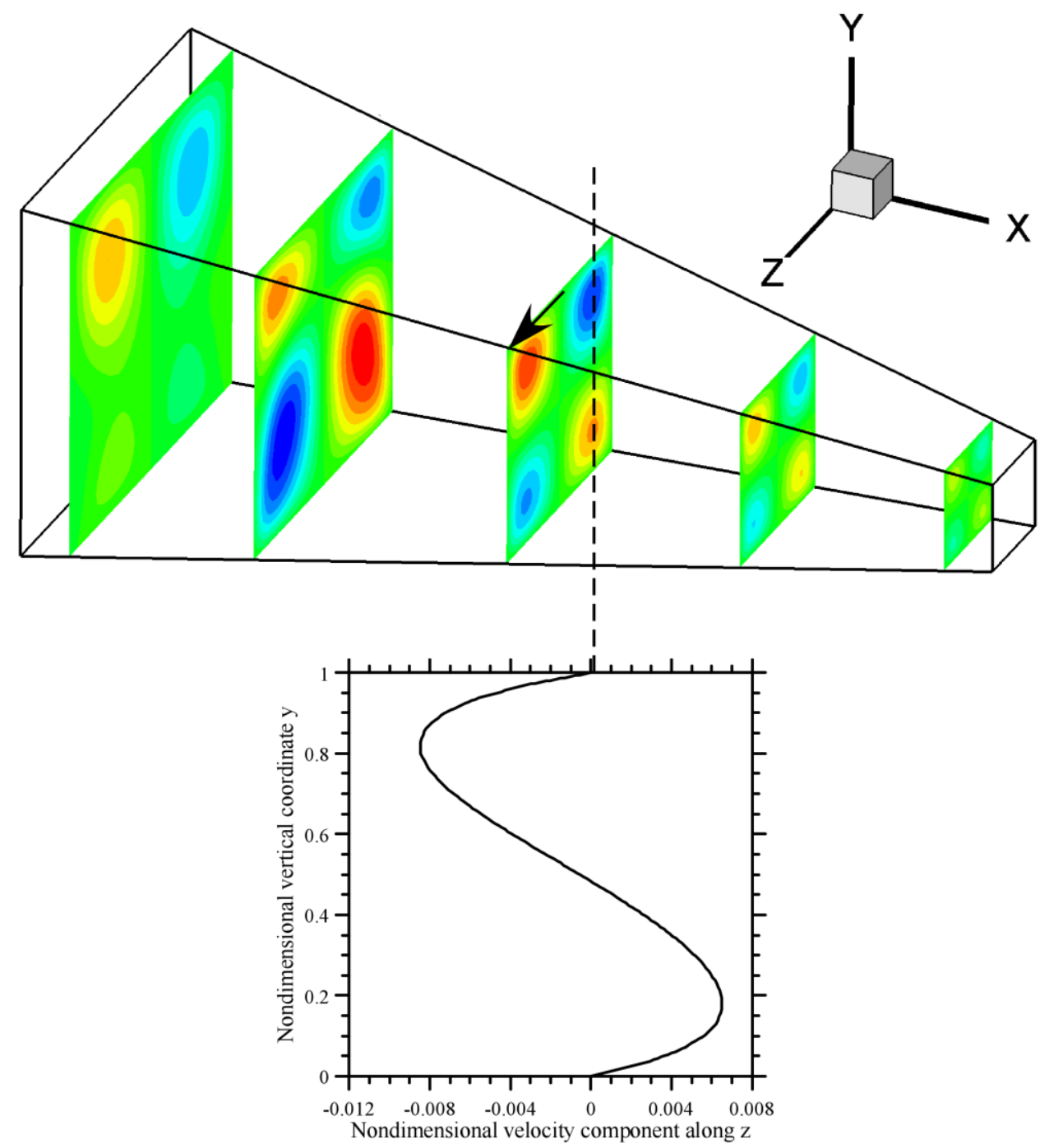

FIG. 8 STEDY VELOCITY FIELD (SLICES OF THE V ELOCITY COMPONENTW)FOR A=4, Ra=100 and $\eta=1 / 16$ (3D SIMULATIONFOR A CONTAINER HAVING THE SHAPE OF A FRUSTUM OF PYRAMID, NxxNy xNz=100x30x30) AND W VELOCITY PROFILE AT x=A/2 AND $\mathrm{z}=1 / 4$.

Given the very small value of the Prandtl number considered ( $\operatorname{Pr}=0.01)$, the simple observations above give rise to the important question to understand whether such 2D or 3D flows could become unstable against the onset of disturbances of hydrodynamic nature. Such instabilities, in the past studied essentially for parallel flows or flows 
in rectangular cavities with finite extent, are known to be driven by the mean shear stress (this is the reason why they are often referred to as "shear instability" and the related disturbances as hydrodynamic ones). As a result, some vortices appear on the frontier of the two opposing (primary and return) horizontal currents characterizing the basic flow. Such disturbances are in general stationary (the general outcome of this instability is the replacement of the initial "unicellular" Hadley flow with a multicellular convective structure) and become oscillatory when a second threshold in terms of applied temperature gradient is exceeded (Gelfgat et al., 1999; Kaddeche et al., 2003).

\section{Conclusions and Future Directions}

The typical structure of the Hadley flow in containers having varying cross-sectional area in the case of liquid metals has been investigated on the basis of a hybrid approach based on the numerical solution of the Navier Stokes equations in conjunction with analytic solutions of the temperature field (used as initial conditions). The analysis has provided heretofore unseen information about the role played by converging or diverging walls on the fundamental properties of the emerging flow in terms of strength, patterning behaviour and the existence of "inflection" points in the velocity profile.

The existence of such points and the non-trivial dependence displayed by the intensity of the flow and the related shear stress on the geometry characteristic parameters call for a "future" analysis specifically conceived to address the potential onset of disturbances of hydrodynamic nature at larger values of the control parameter Ra. Such disturbances are expected to break the typical initial uni-cellular flow via stationary or oscillatory bifurcations and produce significant asymmetries in the dependence of the maximum shear stress and streamfunction on the compression (or expansion) ratio.

\section{REFERENCES}

[1] Abhilash E., Joseph M.A. and Krishna P., "Prediction of Dendritic Parameters and Macro Hardness Variation in Permanent Mould Casting of Al-12\%Si Alloys Using Artificial Neural Networks", Fluid Dyn. Mater. Process., 2(3) (2006): 211-220

[2] Delgado-Buscalioni R. and Crespo del Arco E., "Flow and heat transfer regimes in inclined differentially heated cavities", Int. J. Heat Mass Transfer, 44 (2001): 1947-1962.

[3] Drazin P. and Howard L.N., "Hydrodynamic stability of parallelflow of inviscid fluid", Adv. Appl. Mech., 9 (1966): 1-89.

[4] Dupret F. and Van der Bogaert N., "Modelling Bridgman and Czochralski growth", in Handbook of Crystal Growth (ed. D. T. J.Hurle) 2: 877-1010. North-Holland, Amsterdam (1994).

[5] Fletcher C.A.J.," Computational te chniques for fluid-dynamics", (Springer Verlag, Berlin, 1991).

[6] Gelfgat A.Yu., Bar-Yoseph P.Z. and Yarin A.L., "Stability of Multiple Steady States of Convection in Laterally Heated Cavities", J. Fluid Mech., 388 (1999): 315-334.

[7] Hadley G., "Concerning the cause of the general trade winds", Phil. Trans. Roy. Soc. Lond., 29 (1735): 58-62.

[8] Hong B., Armaly B.F., Chen T.S., "Laminar mixed convection in a duct with a backward-facing step-the effects of inclination angle and prandtl number", Internat. J. Heat Mass Trans. 36 (1993): 3059-3067.

[9] Kaddeche S., Henry D. and Ben Hadid H., "Magnetic stabilization of the buoyant convection between infinite horizontal walls with a horizontal temperature gradient", J. Fluid Mech., 480 (2003): 185-216.

[10] Ladyzhenskaya O.A., "The Mathematical Theory of Viscous Incompressible Flow”, Gordon and Breach, 2nd Edition, New York - London, 1969.

[11] Lappa M., "Thermal convection and related instabilities in models of crystal growth from the melt on earth and in microgravity: Past history and current status", Cryst. Res. Technol., 40(6) (2005): 531-549.

[12] Lappa M., “ThermalConvection: Patterns, Evolution and Stability”, John Wiley \& Sons, Ltd (2009, Chichester, England). 
[13] Lappa M., (2012a), “Exact Solutions for Thermal Problems: Buoyancy, Marangoni, Vibrational and Magnetic-FieldControlled Flows", Review of Applied Physics, 1(1) (2012): 1-14.

[14] Lappa M., (2012b), "Rotating Thermal Flows in Natural and Industrial Processes", John Wiley \& Sons, Ltd (2012, Chichester, England).

[15] Lin C.-C., “On the stability of two-dimensional parallelflows”, Proc. NAS, 30(10) (1944): 316-324.

[16] Ludwig A., Gruber-Pretzler M., Wu M., Kuhn A. and Riedle J., “About the Formation of Macrosegregations During Continuous Casting of Sn-Bronze", Fluid Dyn. Mater. Process., 1(4) (2005): 285-300.

[17] Monberg E., “Bridgman and related growth techniques”. In Handbook of Crystal Growth (ed. D. T. J. Hurle), 2: 53-97. North-Holland, Amsterdam (1994).

[18] Rosenbluth M.N. and Simon A., "Necessary and sufficient conditions for the stability of plane parallel inviscid flow", Phys. Fluids, 7(4) (1964): 557-558.

[19] Tollmien W., "General instability criterion of laminar velocity distributions", Tech. Memor. Nat. Adv. Comm. Aero., Wash. No. 792 (1936).

[20] Zrodnikov A.V., Efanov A.D., Orlov Yu.I., Martinov P.N., Troynov V.M., Rusanov A.E., “Technology of heavy liquid metal coolants lead-bismuth and lead", VANT, Serial: Provision of NPP Safety, Issue 4, Nuclear Technologies for Future Power System, (2004): 180-184. 